\title{
Experiencia y manejo de dos pacientes pediátricos grandes quemados e infección por SARS-CoV-2 en una unidad de cuidados intensivos
}

\section{Experience and management of two paediatric patients infected with SARS-CoV-2 with major burn injuries in an intensive care unit}

Correspondencia Normy De Pawlikowski ndepawlikowski@insnsb.gob.pe

Recibido: 25/08/2020

Arbitrado por pares

Aprobado: 24/09/2020

Citar como: Bustamante A, Miranda J, Iglesias M, Contreras K, Lector C, De Pawlikowski N. Experiencia y manejo de dos pacientes pediátricos grandes quemados e infección por SARS-CoV-2 en una unidad de cuidados intensivos. Acta Med Peru. 2020;37(4):505-10. doi: https://doi. org/10.35663/amp.2020.374.1675
Atenas Bustamante ${ }^{1, a}$, Juan Miranda ${ }^{1, a}$, Martin Iglesias ${ }^{1, a}$, Kelly Contreras ${ }^{1, a}$, Christian Lector ${ }^{1, a}$, Wieslawa De Pawlikowski ${ }^{1, a, b}$

Instituto Nacional de Salud del Niño - San Borja. Lima, Perú

Médico cirujano; ${ }^{b}$ especialista en Cirugía Plástica y Reconstructiva

\section{RESUMEN}

La infección por SARS-CoV-2 se ha diseminado rápidamente, provocando una pandemia mundial en la cual nos vemos afectados. En el Instituto Nacional de Salud del Niño de San Borja, centro de referencia nacional de pacientes pediátricos quirúrgicos, desde el 14 de abril al 12 de agosto del 2020, se hospitalizaron 106 pacientes con infección por SARS-CoV-2, de los cuales 11 tuvieron diagnostico quemadura y dos fueron pacientes grandes quemados con diagnóstico COVID-19 por prueba molecular. Detallamos el caso de una paciente pediátrica, de siete años, gran quemada que fue atendida en unidad de cuidados intensivos, y con COVID-19 asintomática, presentó evolución y pronóstico favorable, con recuperación total de su lesión. Por otro lado, el COVID-19 se puede manifestar de forma severa produciendo un síndrome inflamatorio multisistémico que presenta manifestaciones clínicas poco comunes y que puede empeorar el pronóstico, como fue observado en nuestro otro paciente de siete meses que falleció pese a recibir manejo especializado y oportuno.

Palabras clave: COVID-19; SARS-CoV-2; Quemaduras; Niño (fuente: DeCS BIREME).

\section{ABSTRACT}

SARS-CoV-2 infection is rapidly disseminating, leading to a world pandemics in which we are also affected. In San Borja Peruvian National Children Health Institute, which is the reference center for surgical pediatric patients, 106 patients infected with SARS-CoV- 2 were hospitalized. Of them, 11 presented with burns and two had large burns. The viral infection was diagnosed using molecular testing. We present in detail the case of a pediatric seven-year old patient who had large burns and was taken care of in the intensive care unit. She had asymptomatic SARS-CoV-2 infection, and her outcome was good, with a good prognosis, and she recovered completely and uneventfully. On the other hand, COVID-19 may present as a severe disease leading to a multisystemic inflammatory syndrome with some uncommon clinical manifestations which affect its prognosis, as it was the case with another seven-month old patient who passed away in spite of having received specialized and timely therapy.

Keywords: COVID-19; SRAG-CoV-2; Burns; Child (source: MeSH NLM). 


\section{INTRODUCCIÓN}

En diciembre del año 2019, en Wuhan, China ${ }^{[1]}$ se reportó la primera infección en humanos por Severe Acute Respiratory Syndrome Coronavirus 2 (SARS-CoV-2), así denominado por el Comité Internacional de Taxonomía de Virus ${ }^{[2]}$. Asimismo, la Organización Mundial de la Salud nombró a la enfermedad causada por este virus Coronavirus disease 2019 (COVID-19) ${ }^{[3]}$. Desde entonces, el COVID-19 se ha propagado rápidamente, provocando una pandemia mundial ${ }^{[4]}$.

El cinco de marzo del 2020 se reportó el primer caso de enfermedad por COVID-19 en el Perú y el 11 de marzo se declaró estado de emergencia nacional ${ }^{[5]}$. Desde entonces, al 12 de agosto, en el Perú se detectaron 22836 niños y adolescentes con COVID-19 ${ }^{[6]}$.

En el Instituto Nacional de Salud del Niño de San Borja (INSNSB) el primer caso de COVID-19 ingresó el 13 de abril y el cuatro de mayo se hospitalizó la primera paciente gran quemada con infección por SARS-CoV-2 en la Unidad de Cuidados Intensivos $(\mathrm{UCl})^{[7]}$.

Los pacientes grandes quemados requieren un tratamiento oportuno y eficaz para disminuir la morbimortalidad causada por la injuria térmica. La respuesta inflamatoria sistémica que se produce está relacionada a la magnitud de la quemadura en la cual los fenómenos inflamatorios pueden amplificarse o mantenerse por efectos intercurrentes ${ }^{[8]}$.

La infección por SARS-CoV-2 ha demostrado producir una cascada inflamatoria multisistémica ${ }^{[9]}$ cuyas manifestaciones clínicas varían desde la presencia de síntomas leves hasta una falla respiratoria que puede estar asociada a disfunción multiorgánica ${ }^{[10]}$.

En el INSN-SB se implementaron medidas para el adecuado manejo de los pacientes sospechosos y confirmados de SARSCoV-2, adoptándose protocolos de atención para emergencia y hospitalización ${ }^{[11]}$ (Figura 1).

El presente reporte describe el curso clínico, evolución y tratamiento de dos pacientes pediátricos grandes quemados que presentaron infección por SARS-CoV-2, diagnosticados por prueba molecular y hospitalizados en la UCI pediátrica (UCI PED) del INSN-SB durante el periodo 14 de abril al 12 de agosto 2020. El resumen de las características clínicas y epidemiológicas, así como el manejo clínico y quirúrgico se muestran en las Tablas 1 у 2.

\section{REPORTE DE CASOS}

\section{CASO 1}

Paciente mujer de siete años, de $25 \mathrm{~kg}$, procedente de Lima, sin antecedentes previos relevantes. Dos horas antes del ingreso, sufrió quemadura de II/III grado por fuego directo. Siete horas después del ingreso se realizó curación bajo anestesia en sala de operaciones COVID (SOP COVID), evidenciándose quemadura de $20,5 \%$ superficie corporal total (SCT). Se cubrieron las lesiones en tronco con apósito hidrocoloide y las lesiones en cara con biocelulosa.

La paciente fue hospitalizada en en UCI PED y se solicitó toma de prueba molecular reacción en cadena de la polimerasa con transcriptasa inversa (RT-PCR) para SARS-CoV-2. Recibió hidratación (lactato Ringer $100 \mathrm{~cm}^{3} / \mathrm{h}$ ), antibioticoterapia (oxacilina 1 gramo $\mathrm{c} / 6 \mathrm{~h}$ ), analgesia (ketamina + morfina en infusión continua) y nutrición enteral (formula polimérica $18 \%$ $13 \mathrm{~cm}^{3} / \mathrm{h}$ por 20 horas), no requirió apoyo inotrópico.

Dos días después del ingreso se recibió resultado RT-PCR positivo para SARS-CoV-2; la paciente se encontraba hemodinámicamente estable y se la trasladó a área COVID. Tres días después del ingreso se cambió antibioticoterapia (piperacilina/tazobactam 1,9 gramos $\mathrm{c} / 6 \mathrm{~h}$ ) por aumento de reactantes de fase aguda. Seis días después del ingreso, ingresó a SOP COVID donde se le realizó escarectomía y colocó autoinjerto de espesor parcial en un $8 \%$ de SCT.

Catorce días después del ingreso, fue trasladada nuevamente a SOP COVID para limpieza quirúrgica y autoinjerto de piel parcial en un $5 \%$ SCT. Veinticuatro días posteriores al ingreso se le realizó limpieza quirúrgica y autoinjerto de piel parcial del $2 \%$ SCT en SOP COVID. Veintiocho días después del ingreso, la paciente fue dada de alta sin síntomas respiratorios y evolución clínica quirúrgica favorable.

\section{CASO 2}

Paciente varón de 10 meses, de $8 \mathrm{~kg}$, que fue transferido de la ciudad de Huancavelica, sin antecedentes previos relevantes. Un día antes de su ingreso sufrió quemadura de II/III grado por líquido caliente. Paciente no presentaba sintomatología respiratoria. Seis horas después del ingreso, se le realizó curación bajo anestesia en SOP COVID, evidenciándose quemadura de $26 \%$ SCT. Se le colocaron biocelulosa en lesión facial y apósito de plata a nivel corporal.

El paciente fue hospitalizado en UCI PED y se le realizó prueba molecular RT-PCR. Se instauró tratamiento antibiótico (oxacilina $465 \mathrm{mg} \mathrm{c} / 6 \mathrm{~h}$ ), hidratación (lactato Ringer $30 \mathrm{~cm}^{3} / \mathrm{h}$ ), nutrición (fórmula maternizada al $13 \%$ por sonda nasogástrica) y analgesia endovenosa (ketamina + morfina en infusión continua), no requiriendo apoyo inotrópico.

Cuatro días después del ingreso, a la auscultación, el paciente presentaba ruidos "roncantes" en ambos campos pulmonares y requirió uso de oxígeno por cánula binasal con $\mathrm{FiO}_{2}$ al $32 \%$. En la radiografía de tórax se evidenciaban opacidades alveolares parcheadas difusas en ambos campos pulmonares (Figura 2B). Al quinto día de hospitalización, se vio una involución clínica, 


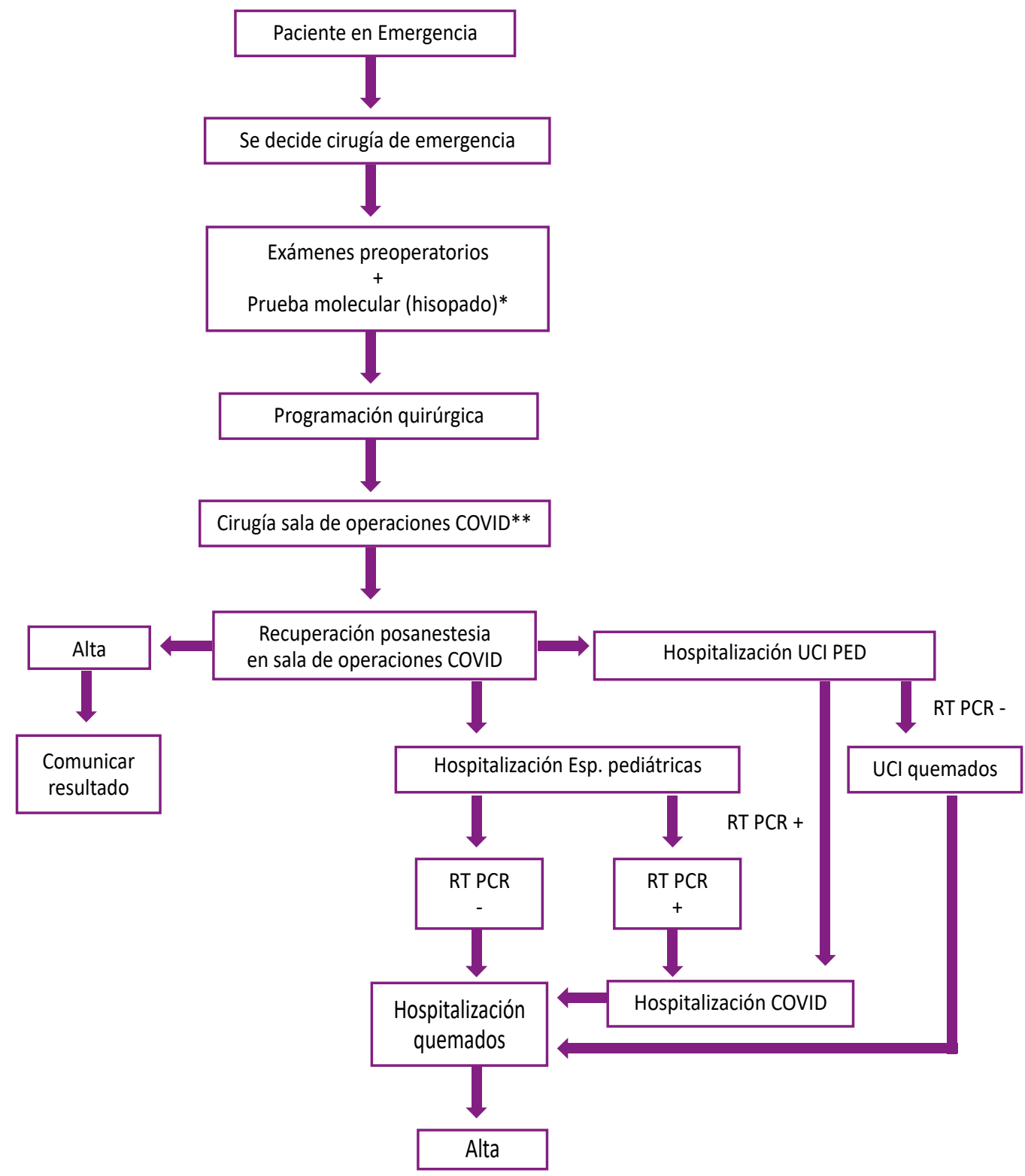

UCI: unidad de cuidados intensivos; UCI PED: UCI pediátrica; RT PCR: reacción en cadena de la polimerasa con transcriptasa inversa; Esp. pediátricas: sala de Especialidades pediátricas.

Fuente: Propuesta de flujograma para manejo quirúrgico de cirugía electiva - especialidades quirúrgicas. Instituto Nacional de Salud del Niño San Borja. Lima, 5 de Julio del 2020.

Figura 1. Flujorama para el manejo de pacientes quirúrgicos atendidos en emergencia del Instituto Nacional de Salud del Niño de San Borja Modificado.

presentando insuficiencia respiratoria. Por tal motivo, fue intubado, evidenciándose neumotórax a tensión en radiografía (Figura 2C).

Posteriormente, el paciente presentó desaturación severa y paro cardiorrespiratorio, por lo que recibió reanimación cardiopulmonar avanzada durante 20 minutos, sin respuesta. Se constató fallecimiento de paciente. Se obtuvo resultado de prueba molecular RT-PCR COVID-19 positivo un día luego del fallecimiento.

\section{DISCUSIÓN}

En el INSN-SB, centro quirúrgico pediátrico de referencia a nivel nacional, se hospitalizaron 5368 pacientes durante el año 2019, de los cuales 516 tuvieron el diagnóstico de quemadura ${ }^{[12]}$. En pacientes pediátricos, el $23 \%$ de aquellos con el diagnóstico de quemadura requirieron un manejo en $\mathrm{UCl}^{[13]}$. Del $14 \mathrm{de}$ abril al 12 de agosto 2020, se hospitalizaron 106 pacientes con COVID-19 y 31 fueron atendidos en la UCI PED. Once pacientes 
Tabla 1. Características epidemiológicas y clínicas de dos pacientes grandes quemados pediátricos con COVID-19 hospitalizados en la UCl pediátrica.

\begin{tabular}{|c|c|c|}
\hline Características & Caso 1 & Caso 2 \\
\hline Sexo & $\mathrm{F}$ & M \\
\hline Edad & 7 años & 10 meses \\
\hline SCTQ & $20 \%$ & $26 \%$ \\
\hline Agente & fuego directo & líquido caliente \\
\hline Comorbilidades & ninguna & ninguna \\
\hline Síntomas respiratorios & ninguno & ninguno \\
\hline Contacto con persona infectada Covid-19 & ninguno & ninguno \\
\hline Diagnóstico de COVID-19 & RT-PCR & RT-PCR \\
\hline Estancia en UCl & 4 días & 5 días \\
\hline Estancia hospitalaria & 28 & 5 días \\
\hline $\begin{array}{l}\text { Tiempo transcurrido desde quemadura hasta ingreso a } \\
\text { INSN-SB }\end{array}$ & 2 horas & 32 horas \\
\hline Sobrevida & Sí & No \\
\hline
\end{tabular}

SCTQ: superficie corporal total quemada; UCI: unidad de cuidados intensivos; PR: prueba rápida para detección de anticuerpos contra SARS-CoV-2; RT-PCR: reacción en cadena de la polimerasa con transcriptasa inversa para detección de SARS-CoV-2.

tuvieron diagnóstico de quemadura y COVID-19 y dos de ellos, con diagnóstico por prueba molecular, recibieron tratamiento en la UCI PED ${ }^{[14]}$.

Para adaptarse al COVID-19, el INSN-SB adoptó un protocolo de atención (Figura 1) que indicaba que a todo paciente quirúrgico que ingresase por emergencia debía realizársele una prueba molecular de RT-PCR, exámenes auxiliares de laboratorio, radiografía de tórax y catalogársele como caso sospechoso. La curación se realiza SOP COVID y el paciente es hospitalizado en UCI PED, en espera de resultados de RT-PCR procesados por el Instituto Nacional de Salud, pasando posteriormente a UCI quemados si el resultado de RT-PCR fuese negativo.
El manejo de la quemadura se realiza siguiendo el protocolo de nuestra institución y se enfoca en cinco esferas: hidratación inmediata, estado hemodinámico, dolor, antibioticoterapia y nutrición ${ }^{[13]}$. Se realizan curaciones espaciadas entre tres y cinco días y, en casos necesarios, cirugías e injertos precoces en SOP COVID. Dichas medidas concuerdan con las experiencias reportadas en otros centros hospitalarios especializados en quemados a nivel mundial ${ }^{[15-18]}$.

Según los Centers for Disease Control and Prevention de Estados Unidos, los grupos de niños y adolescentes representaron el 1,7\% de los casos diagnosticados con COVID-19, en su territorio ${ }^{[19]}$; mientras que el Ministerio de Salud del Perú informó que la

Tabla 2. Flujo de atención de siguieron pacientes grandes quemados COVID según flujograma del INSN SB modificado.

\begin{tabular}{lcc}
\multicolumn{1}{c}{ Variable } & Caso 1 & Caso 2 \\
\hline Tipo de nutrición & SNG & SNG \\
Apoyo ventilatorio & So & Sí \\
Tratamiento antibiótico recibido & Piperacilina-Tazobactam + Oxacilina & Oxacilina/ Vancomicina + \\
SCTQ & $20 \%$ & Meropenem \\
SCTQ injertada & $15 \%$ & $26 \%$ \\
$\mathrm{~N}^{\circ}$ de intervenciones quirúrgicas & 1 & $0 \%$ \\
Tratamiento de Quemadura & Escarectomía + Autoinjerto & 0 \\
\hline
\end{tabular}

SCTQ: superficie corporal total quemada; STP: sonda transpilórica; SNG: sonda nasogástrica 


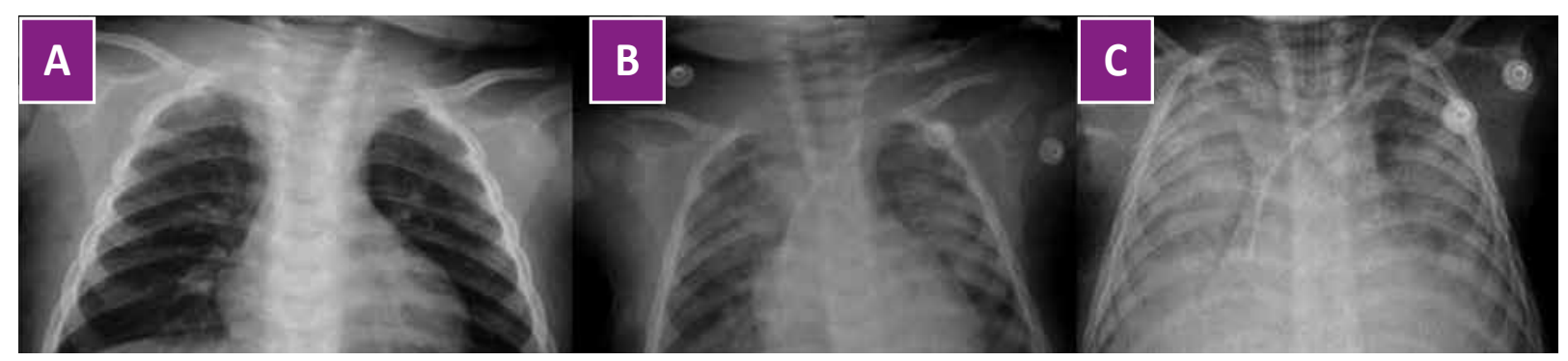

Fuente: Programa PAC-S del Instituto Nacional de Salud del Niño - San Borja.

Figura 2. Progresión radiológica del caso 2. A. Radiografía de tórax al ingreso: discreto engrosamiento bronquial parahiliar. B. Radiografía de tórax cuatro días después del ingreso: opacidades alveolares parcheadas difusas en ambos campos pulmonares. $\mathbf{C}$. Radiografía de tórax cinco días después del ingreso: opacidades alveolares parcheadas difusas en ambos campos pulmonares en mayor proporción que el día previo.

población pediátrica representaba el $4,7 \%$ del total de casos diagnosticados ${ }^{[6]}$.

Estudios iniciales reportaban que los niños con COVID-19 tienen una evolución clínica asociada a menor sintomatología y manifestaciones clínico- radiológicas que la población adulta ${ }^{[20]}$, y hasta el 35\% de los niños con COVID-19 podrían ser asintomáticos ${ }^{[21]}$. En nuestro reporte, el COVID-19 se manifestó de forma asintomática en uno de nuestros pacientes y según lo observado, no influyó en el curso clínico de la quemadura.

El COVID-19 se puede manifestar de forma severa en la población pediátrica produciendo un síndrome inflamatorio multisistémico (Paediatric Inflammatory Multisystem Syndrome Temporally Associated with SARS-CoV-2) ${ }^{[22]}$. Es posible que la cascada inflamatoria multisistémica exagerada provocada por el SARSCoV-2 puede comprometer la evolución clínica de los pacientes grandes quemados, donde la injuria térmica produce liberación de mediadores inflamatorios que conlleva un alto grado de morbimortalidad ${ }^{[23]}$. Como se describió anteriormente, uno de nuestros pacientes presentó clínica severa de COVID-19, padeció una insuficiencia respiratoria que empeoró la evolución clínica de la quemadura y falleció siete días después de su ingreso, pese al adecuado manejo de la quemadura. Esta evolución es asemeja el caso reportado por Toh et al., en el que una paciente con quemadura del $50 \%$ SCT presentó, al cuarto día de ingreso hospitalario, compromiso respiratorio compatible con COVID-19 y trombosis venosa profunda, falleciendo tres días después ${ }^{[24]}$.

En este reporte observamos que uno de nuestros pacientes fue asintomático/leve, presentó evolución y pronóstico favorable, con recuperación total de su lesión. No obstante, el COVID-19 se puede manifestar de forma severa produciendo un síndrome inflamatorio multisistémico y empeorar el pronóstico, como se observó en nuestro otro paciente, a pesar de manejo especializado y oportuno.

Este trabajo es uno de los primeros que describe el curso clínico, evolución y tratamiento de pacientes pediátricos grandes quemados diagnosticados con COVID-19, esperamos que sirva como base para estudios futuros que se enfoquen en este tema, evaluando si el fenómeno inflamatorio que se desencadena a consecuencia de la infección por SARS-CoV-2 pudiese potenciar la respuesta inflamatoria multisistémica que ocurre posterior a una quemadura extensa en pacientes pediátricos, agravando de esta manera, el curso clínico de la quemadura.

Contribuciones de autoría: $\mathrm{NdP}, \mathrm{MI}, \mathrm{AB}$ y JP realizaron la recolección de los datos. $\mathrm{NdP}, \mathrm{MI}$ y $\mathrm{AB}$ contribuyeron con la redacción y revisión crítica del manuscrito. Todos los autores realizaron el diseño del manuscrito, análisis e interpretación de los datos y aprobación de la versión final a publicarse.

\section{Fuente de financiamiento: Autofinanciado.}

Potencial conflicto de intereses: Los autores declaran no tener conflictos de intereses.

\section{ORCID}

Normy De Pawlikowski, https://orcid.org/0000-0003-3331-1166 Martin Iglesias, https://orcid.org/0000-0002-2799-7483 Atenas Bustamante, https://orcid.org/0000-0003-4836-0761 Juan Miranda, https://orcid.org/0000-0002-6209-3885 Kelly Contreras, https://orcid.org/0000-0003-3513-5397 Christian Lector, https://orcid.org/0000-0003-1123-8519

\section{REFERENCIAS BIBLIOGRÁFICAS}

1. Gorbalenya AE, Baker SC, Baric RS, de Groot RJ, Drosten C, Gulyaeva $A A$, et al. Severe acute respiratory syndrome-related coronavirus: the species and its viruses-a statement of the Coronavirus Study Group. BioRxiv. 2020:3-12.

2. Outbreak of acute respiratory síndrome associated with a novel coronavirus, Wuhan, China; first update. 22 January 2020 [Internet]. Stockholm: ECDC; 2020 [citado 24 marzo 2020]. Disponible en: https://www.ecdc.europa.eu/sites/default/files/documents/Riskassessment-pneumonia-Wuhan-China-22-Jan-2020.pdf 
3. Lai CC, Shih TP, Ko WC, Tang HJ, Hsueh PR. Severe acute respiratory syndrome coronavirus 2 (SARS-CoV-2) and coronavirus disease-2019 (COVID-19): the epidemic and the challenges. Int J Antimicrob Agents. 2020;105924. doi: 10.1016/j.ijantimicag.2020.105924.

4. WHO. Coronavirus disease 2019 (COVID-19) Situation Report 63, World Health Organization [Internet]. Ginebra: WHO; 2020 [citado 24 marzo 2020]. Disponible en: https://apps.who.int/iris/ bitstream/handle/10665/331607/nCoVsitrep23Mar2020-eng. pdf?sequence=1\&isAllowed=y

5. Ministerio de Salud del Perú. Resolución Ministerial º89-2020. Plan de Estrategia Publicitaria 2020 del Ministerio de Salud. Lima: MINSA; 2020.

6. Ministerio de Salud del Perú. Vigilancia, Prevención y Control del COVID-19. Situación Actual “COVID-19" Perú - 2020, 09 de agosto [Internet]. Lima: Dirección General de Epidemiología, MINSA; 2020 [citado 13 agosto 2020]. Disponible en: https://www.dge.gob.pe/ portal/docs/tools/coronavirus/coronavirus090820.pdf

7. Instituto Nacional de Salud del Niño de San Borja. INSN-SB Blog [Internet]. Lima; INSN SB; 2020 [citado 28 junio 2020]. Disponible en: http://www.insnsb.gob.pe/blog/category/mayo/

8. Ravat F, Payre J, Peslages P, Fontaine M, Sens N. La brûlure : une pathologie inflammatoire [Burn: An inflammatory process]. Pathol Biol (Paris). 2011;59(3):e63-72. doi: 10.1016/j.patbio.2009.12.001

9. Zhou Y, Fu B, Zheng $X$, Wang $D$, Zhao $C$, qi Y, et al. Pathogenic T cells and inflammatory monocytes incite inflammatory storm in severe COVID-19 patients. Natl Sci Rev. 2020;7(6):998-1002. doi: 10.1093/ nsr/nwaa041.

10. Yuki K, Fujiogi M, Koutsogiannaki S. COVID-19 pathophysiology: A review Clin Immunol. 2020 Jun; 215: 108427. doi: 10.1016/j. clim.2020.108427.

11. Instituto Nacional de Salud del Niño San Borja. Informe $N^{\circ} 000106$ 2020-DA-DG-INSNSB. Propuesta de flujograma para manejo quirúrgico de cirugía electiva - especialidades quirúrgicas. 5 de Julio del 2020. Lima: INSN SB; 2020.

12. Instituto Nacional de Salud del Niño de San Borja. Estadística de número de pacientes con diagnóstico de quemadura atendidos en el año 2019. Lima: INSN-SB; 2019.

13. Huby Vidaurre M del P. Quemaduras pediátricas en el Perú. Diagnostico. 2017;56(4):185-9.
14. Instituto Nacional de Salud del Niño San Borja. Sala situacional COVID de los pacientes hospitalizados en el INSN-SB al 12 agosto. Lima: INSN SB; 2020.

15. Huang Z, Zhuang D, Xiong B, Deng DX, Li H, Lai W. Occupational exposure to SARS-CoV-2 in burns treatment during the COVID-19 epidemic: Specific diagnosis and treatment protocol. Biomed Pharmacother. 2020;127:110176. doi: 10.1016/j. biopha.2020.110176

16. Ma S, Yuan Z, Peng Y, Chen J, Li H, Luo Q, et al. Experience and suggestion of medical practices for burns during the outbreak of COVID-19. Burns. 2020;46(4):749-55. doi: 10.1016/j. burns.2020.03.014.

17. Li N, Liu T, Chen H, Liao J, Luo Q, Song H, et al. Management strategies for the burn ward during COVID-19 pandemic. Burns. 2020;46(4):756-61. doi: 10.1016/j.burns.2020.03.013.

18. Barret JP, Chong SJ, Depetris N, Fisher MD, Luo G, Moiemen N, et al. Burn center function during the COVID-19 pandemic: An international multi-center report of strategy and experience. Burns. 2020;46(5):1021-35. doi: 10.1016/j.burns.2020.04.003.

19. CDC COVID-19 response team. Coronavirus disease 2019 in Children - United States, February 12-April 2, 2020. MMWR Morb Mortal Wkly Rep. 2020;69(14):422-426. doi: 10.15585/mmwr.mm6914e4.

20. Zimmermann P, Curtis N. COVID-19 in children, pregnancy and neonates: a review of epidemiologic and clinical features. Pediatr Infect Dis J. 2020;39:469-77. doi: 10.1097/INF.0000000000002700.

21. Tang A, Xu W, Shen M, Chen P, Li G, Liu Y, et al. A retrospective study of the clinical characteristics of COVID-19 infection in 26 children. medRxiv. 2020. doi: 10.1101/2020.03.08.20029710.

22. Royal College of Paediatrics and Child Health. GuidancePaediatric multisystem inflammatory syndrome temporally associated with COVID-19 [Internet]. RCPCH; 2020 [citado 5 mayo 2020]. Disponible en: https://www.rcpch.ac.uk/sites/ default/files/2020-05/COVID-19-Paediatric-multisystem-\%20 inflammatory\%20syndrome-20200501.pdf

23. Krishnamoorthy V, Ramaiah R, Bhananker SM. Pediatric burn injuries. Int J Crit IIIn Inj Sci. 2012;2(3):128-34. doi: 10.4103/22295151.100889 .

24. Toh VV, Antrum JHG, Sloan B, Austin O, Muthayya P. Management of COVID-19 in burns patients: The experience of a UK burn centre. Burns. 2020;46(7):1710-2. doi: 10.1016/j.burns.2020.06.026. 